\title{
A New Generation of Evaluation Tool for On-Line Design and Scheduling in An Advanced Manufacturing System
}

\author{
Ming-Hung Lin and Li-Chen Fu \\ Dept. of Computer Science and Information Engineering \\ National Taiwan University,Taipei,Taiwan,R.O.C \\ E-mail: lichen@ccms.ntu.edu.tw
}

\begin{abstract}
In business, new product development is the name of the game. An efficient evaluation tool is crucial. This paper proposes a new generation of evaluation tool for on-line design and scheduling, wherein planned control policy designed by engineers will be on-line evaluated. Performance will be evaluated by a new prediction rule that differs from existent stateindependent or steady state model such as a queuing network model. In addition to that, a new optimal control rule or schedule can then be derived from the original planned ones and the feedback from the manufacturing engineers. Simulation and prediction methods are applied together to give an optimum in the timeslot of snapshot. Such evaluation tool can also be used to look for the possible conflicts in the potential solution and suggest some necessary steps to avoid those conflicts.
\end{abstract}

\section{Introduction}

A manufacturing system in today's environment usually has thousands of interrelated variables. The manufacturing systems need not only some negative feedback to keep the enterprise stable when there is a market for them but also some positive feedback to make the systems adaptable to the market. Traditional flexible manufacturing systems - management and evaluation methods based on hierarchical control [6] - are unable to meet the requirements in such a complex environment. Instead, we need a new evaluation support system based on self-organization principle, i.e., an interactive system with self-learning and self-adaptive capabilities.

The rule for measuring the performance is called performance prediction rule. In general, consideration of performance assignment is an important element in the life cycle of production. The queuing theory has been taken as the starting point for such prediction rules [1] [2] [3]. However, queuing network analyzer can only give the average performance over a relatively long period of time. But the performance of manufacturing nowadays usually focuses on a short term to quickly respond to the drastically changing market place. Owing to this, a new prediction rule is needed which differs from existent stateindependent or steady state model such as a queuing network model.

As we all know, most of the scheduling, planning, or layout problem, for example, will require resolution by employing such kind of algorithms. However, if the criteria is represented in analytic form, then the problems at hand will become NP-hard problem. This situation can be alleviated by means of simulation. The joint use of simulation models and optimization methods has been widely studied [4]. Those methods are in the use of the data obtained from the previous simulation to find a new starting point that is close to the global optimum. However, it is not fast enough to meet the changing speed of environment. The area about performance prediction supporting decision making in job-shop production has been proposed [7], but it was limited and just gave conceptual description. The originality of the method in the article is to make use of the data generated by prediction as the direction to find the optimum. According to that, it will be very efficient to respond to the change of the manufacturing environment.

Nowadays, in the complicated real word, it is important to consider more than one objective simultaneously when engineers making decision due to the surrounding complexity [5]. However, the objectives of interest are usually in conflict with one another. Although one objective can be improved, other objectives might be degraded a lot. We proposed a method called negotiation net to support engineers to integrate various kinds of objectives objectives and information effectively. This method also finds the possible conflicts in the potential solution and some necessary steps to avoid the conflicts.

The organization of the paper is as follows. In the next section, we will state the problem and give some discussions. In section 3 , an on-line operation model will be proposed, which will deduce a general approach 


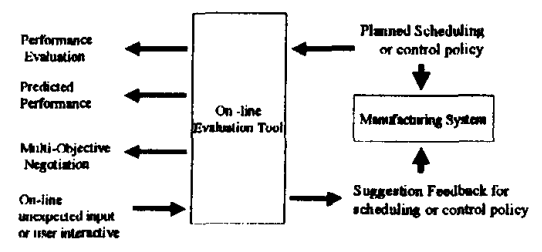

Figure 1: The evaluation tool

for the proposed evaluation tool. Besides that, the prediction diagnosis together with the optimal feedback using simulation and prediction will also be addressed. In section 4 , the proposed evaluation tool is implemented based on a robotic flexible assembly cell, where the proposed tool is tested via a number of simulation. In section 5 , a brief conclusion is made.

\section{Problem Statement and Analysis}

The evaluation tool is a complete developing environment shown in Fig. 1, that was defined to solve the following questions:

(1) How to obtain a better solution derived from the original planned one and to have on-line feedback to manufacturing engineers.

(2) How to measure performance and to give an optimal performance evaluation or prediction, and how to support engineers for evaluation so that it is to integrate many engineers' objectives and information effectively.

\subsection{Performance Prediction}

Given a set of classes of jobs, let $M$ be the total number of classes and $N_{i}$ be the number of job for the $i$ th class. We denote the $j$ th job in the $i$ th class as $j o b_{i j}$. The rule of performance measure is called performance prediction rule, and the consideration of performance assignment is an important element in the life cycle of production. Let $P_{t}$ be a prediction rule generated at time $t$, then the state-dependent prediction process can be formulated as follows:

$$
P_{t}=Z\left[X(t), P_{t^{-}}\right],
$$

where $P_{t^{-}}$is the precedence of the prediction rule at time $t^{-}, X(t)$ is the state variable at time $t$, and $Z$ is denoted as a prediction diagnosis function. Let $D_{i j q}^{R}\left(P_{t}\right)$ be the $q$ th predicted performance of job $i j$ with control policy $R$, and control policy $R$ is to be evaluated with the proposed evaluation tool . Let the $q$ th performance of $j o b_{i j}$ at the end be denoted as
$C_{i j q}(R)$, then our objective is to minimize the rootsquare value of variation $V_{R}^{q}\left(P_{t}\right)$ for the $q$ th predicted performance defined as

$$
V_{R}^{q}\left(P_{t}\right)=\left[\sum_{i j}\left(C_{i j q}(R)-D_{i j q}^{R}\left(P_{t}\right)\right)^{2}\right]^{\frac{1}{2}}
$$

and $V_{R}^{q}\left(P_{t}\right)$ is also denoted as the prediction variation of $P_{t}$.

\subsection{Feedback for Control Policy}

Let $R_{a c k}$ be a new control policy generated by the evaluation tool. Consider the ratio $U(R)$, which is defined as

$$
\begin{gathered}
U_{q}(R)=\prod_{i j} \frac{C_{i j q}(R)}{C_{i j q}\left(R_{a c k}\right)}, C_{i j q}(R) \leq C_{i j q}\left(R_{a c k}\right) \\
U_{q}(R)=\prod_{i j} \frac{C_{i j q}\left(R_{a c k}\right)}{C_{i j q}(R)}, C_{i j q}(R) \geq C_{i j q}\left(R_{a c k}\right),
\end{gathered}
$$

where the control policy $R$ is the original planned one to be evaluated, $q$ is denoted as performance, e.g., throughput, tardiness, and $U_{q}(R)$ is also denoted as the gain of feedback. The problem of feedback for control policy can be mathematically stated as:

$$
\max _{q} U_{q}(R) \longrightarrow \min
$$

subject to

$$
\begin{gathered}
R_{a c k} \in H_{R} \\
H_{R}=\left\{R_{a c k} \mid g_{i}\left(R_{a c k}\right) \leq f_{i}, I=1,2, \cdots, s\right\},
\end{gathered}
$$

where $g_{i}$ and $f_{i}$ are constraints. The objective is then to find a $R_{a c k}$ belonging to the set of admissible solution $H_{R}$ which minimizes the criteria $U_{q}(R)$. The optimization conduction problem $F$ can be formulated as follows:

$$
F\left(R_{a c k}, Y, P_{o}\right) \longrightarrow \min ,
$$

where we define $P_{o}$ as an optimal prediction rule such that $W\left(V_{R}^{q}\left(P_{o}\right)\right)$ is a minimization and $W($.$) is the ex-$ pected value operator. $Y$ is a set of simulation data, $Y=E\left(\bigcup_{i j} j o b_{i j}\right)$, and $E$ is an approximated input function, i.e., random distribution, normal distribution.

\subsection{Multiple Objective}

For easy illustration, considering the due-date assignment $D=\alpha+\beta P+\gamma N$, where $\alpha, D, P, N$ are the arrival time, assigned due-date, total processingtime and the required number of operations of a job, respectively; $\beta$ and $\gamma$ are the respective processingtime and the number of operation multiples. Then, $D$ 
is the objective which will be considered, and we denote $\alpha, P, N$ as characteristic variables $(C V)$. Let $D$ be denoted as the objective of requirement $(O R)$. In general, there is a relationship matrix $M$ which illustrates the relationship between every characteristic variable and every objective of requirement. Suppose we know that $C V \mathrm{~s}$ have effect on every $O R$. The data $M_{i j}$ in the relationship matrix stands for a certain contribution of $C V_{i}$ to $O R_{j}$. From a different viewpoint, data $M_{i j}$ means the probability of a certain $C V$ 's contribution to the satisfaction of a certain $O R_{j}$. No matter what, the value of $M_{i j}$ may be positive or negative, which means that $C V$ s will obstruct or increase a certain $O R_{i}$. The value $M_{i j}$ is also a common random variable, which is a well known distribution with a mean value $m_{i j}$. Let $R$ be a planned control policy to be evaluated by the evaluation tool. We denote the impact function of characteristic variables as $\boldsymbol{\Phi}_{i}$, $C V_{i}=\Phi_{i}(R)$, which means the value of $C V_{i}$ is affected by $R$. Thus, a multi-objective optimization problem can be formulated as

$$
f(R)=\left(f_{O R_{1}}(R), f_{O R_{2}}(R), \cdots, f_{f_{O R_{N}}}(R)\right)
$$

where

$$
f_{O R_{j}}(R)=\sum_{i} M_{i j} \Phi_{i}(R) .
$$

Here, $R^{*}$ is called a completed feasible solution if there exists no other feasible solution $R \in D_{R}$ such that $f_{O R_{j}}(R) \leq f_{O R_{j}}\left(R^{*}\right)$ for all $i=1, \cdots, N$. Consider an associated multi-objective problem from $f(R)$ for a list of weighting parameter $\alpha_{1}, \cdots, \alpha_{N}$ :

$$
\max _{R \in D_{R}} f(R)=\sum_{i} \alpha_{i} f_{O R_{i}}(R)
$$

where $\sum_{i} \alpha_{i}=1$. Then, our goal is to verify $M_{i j}$ to avoid conflict solution, to decide which $\alpha_{i}$ should be urgently improved and to maximize the Equation (7).

\section{The On-line Operation Model of Evaluation Tool}

\subsection{The On-line Operation Model}

The block diagram of on-line operation model for proposed evaluation tool can be presented in Fig. 2 . The components of the on-line operation model are described in the following.

- Manufacturing Monitor Module: This is an entity that is to monitor the status of tasks or machines. This module gets the manufacturing information from the Manufacturing Information Base.

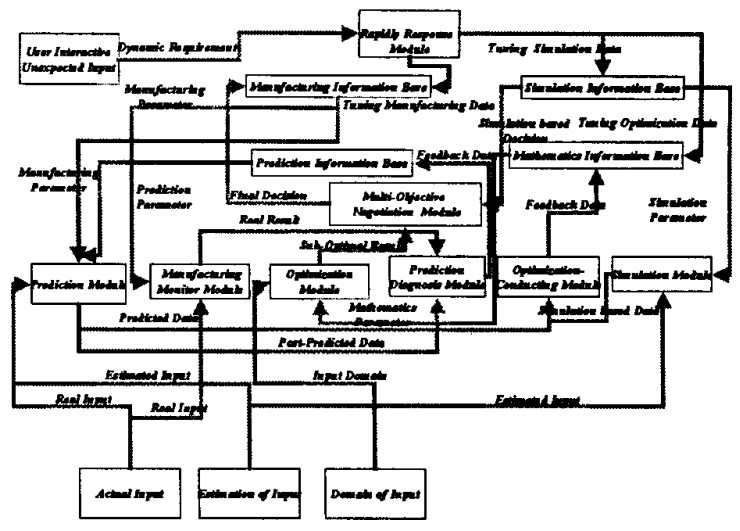

Figure 2: The block diagram of on-line operation model

- Rapidly Response Module: This module handles on-line data such as the interaction from users or unexpected inputs from the environment. It will take the snapshot of on-line manufacturing data; all of prediction module, optimization module and simulation module depend on the snapshot of on-line data.

- Prediction Module: This module takes over the performance evaluation. It has two inputs that are actual on-line manufacturing data and approximated manufacturing data. There are two types of performance evaluation, namely, predicted data and post-predicted data. If the input data are approximated, then the results of performance evaluation belong to predicted data. On the other hand, if the input data are actually manufacturing data, then the results belong to post-predicted data whiles using the same prediction rule. This module takes the current manufacturing parameter from Manufacturing Information Base and parameter of prediction rule from Prediction Information Base.

- Optimization Module: In this module, the criteria of manufacturing problem is represented in analytic form. An optimal solution will be obtained derived from the original planned scheduling or control policies. This module takes the control parameter from Mathematics Information Base.

- Simulation Module: This module performs the simulation before each stage of planned control policy is issued. This module takes the simulation parameter from Simulation Information Base. 


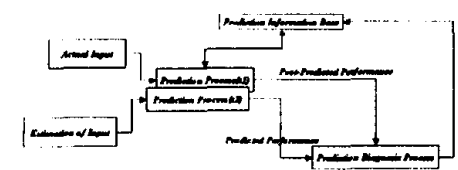

Figure 3: The diagnosis of prediction rule

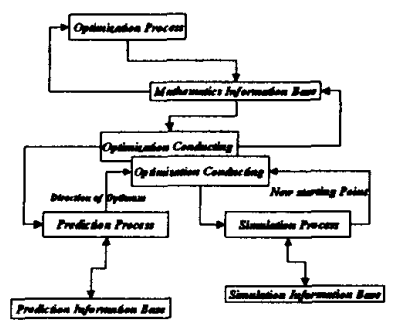

Figure 4: The use of simulation and prediction for optimization

- Prediction Diagnosis Module: The process of prediction diagnosis module is presented generally in Fig. 3. The details of this process can be described in sub-section 3.2 .

- Optimization Conducting Module: The process of optimization conducting module is presented generally in Fig. 4. The details of this process can be described in sub-section 3.3 .

- Multi-objective Negotiation Module: The process of multi-objective negotiation module is presented generally in Fig. 5. The details of this process can be described in sub-section 3.4 .

\subsection{General Approach for Operation}

Although we can obtain an optimal solution for our proposed evaluation tool, it is only for a static and

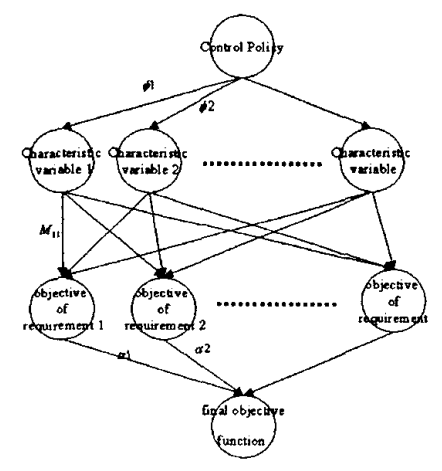

Figure 5: The negotiation network of multi-objective function

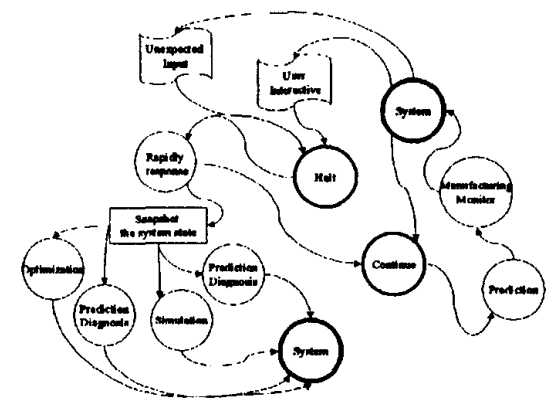

Figure 6: General approach for operation

deterministic one based on the current information. However, the manufacturing environment changes dynamically. One simple rescheduling policy is to perform the optimization module periodically. However, when any stochastic event occurs with the period, it is needed to respond to that situation. Thus, a snapshot is used to report all current manufacturing information, and the Rapidly Response Module will determine what is the better timeslot of this snapshot. The approach of operation can be presented in Fig. 6, whose details can be described in the following :

(1) First, the model is at System state. If there is not any unexpected input or interaction from users, the model enters into the Continue state; otherwise, it enters into the Halt state. If the model is at the Halt state, the Rapidly Response Module will be performed immediately. It determines what is the better timeslot of the snapshot and decides which is the next process. Go to $\operatorname{step}(3)$.

(2) If the model is at the Continue state, the Prediction Module and Manufacturing Monitor Module will be executed. Go to step(5).

(3) If the model does not have to respond to exterior events, the state of the model is set to the Continue state. Go to step (2). Otherwise, perform accurate module properly.

(4) If the module is completed, the state of the model is set to the System state. Go to step (1).

(5) The state of the model is set to the System state. Go to step (1).

The objective of a prediction diagnosis is to minimize equation (2). First, the following initializations are made before we proceed to a detailed analysis of performance prediction:

(a) Jobs arrive at the manufacturing system randomly and follow a well-known static process. The processing-times for each machine are common random variables, which are well known distributions with certain mean value. 
(b) The jobs scheduling is guided by a planned control policy or schedule $R$ that is being evaluated, and the routing of the jobs through the machines are with a fixed probability.

Let the prediction rule $P_{0}$ follows the elementary queuing theory with some tuning variables. With using the result from elementary queuing theory and techniques of the Laplace transforms, the waiting time and flow-time will be approximated as $E_{0}(W)$ and $E_{0}(F)$, respectively, and the approximated prediction of performance can be calculated accordingly.

The prediction-diagnosis process coordinates with the approach of model operation for each run $i$, and the algorithm of prediction-diagnosis process can be summarized in the following steps:

(1) Use Prediction rule $P_{i}$ to approximates $E_{i}(W)$ and $E_{i}(F)$. The new actual Post $E_{i}(W)$ and $\operatorname{Post}_{i}(F)$ will be calculated, where $\operatorname{Post}_{i}(W)$ and $P$ Post $E_{i}(F)$ differ from $E_{i}(W)$ and $E_{i}(F)$, by that they are post-predicted data.

(2) Examine $P_{i}$ subject to equation (2) in this timeslot of snapshot. Form the new $P_{i+1}$ with reference to the performance of post-prediction according to equation (2).

\subsection{Optimization Conducting Process}

The algorithm of optimization conducting process can be summarized in the following steps.

(1) Simulate $\mathrm{N}$ inputs using approximated values. Use the idea of the $\psi$-transform and $\xi$ method proposed by Chichinadze(1969) subject to minimization of the equation (3) and (4), and let $R_{a c k}^{*}$ be the estimation of optimal control policy $R_{a c k}$.

(2) If the estimation error is large, then go back to step (1). Find all of neighbors $R_{a c k}^{* *}$ of $R_{a c k}^{*}$. Coordinate with each run $i$ of prediction diagnosis process, and find the best $R_{a c k}^{* *}$ such that the value of $V_{R_{a c k}^{* *}}^{q}\left(P_{i}\right)$ is the minimum. Replace $R_{a c k}^{*}$ with $R_{a c k}^{* *}$.

Notice that the step (1) consists of running of local optimization calculating several times, and step (2) is a heuristic method in order to forecast the domain of solutions, and to make use of the data generated by prediction as the direction to find the optimum.

\subsection{Multi-objective Negotiation Process}

First, we introduce a relational network called negotiation network. The negotiation network is presented in Fig. 5. Its nodes composed of planned control policy $R$ being evaluated, characteristic variable $(C V)$, objective of requirement $O R$ and final objective function

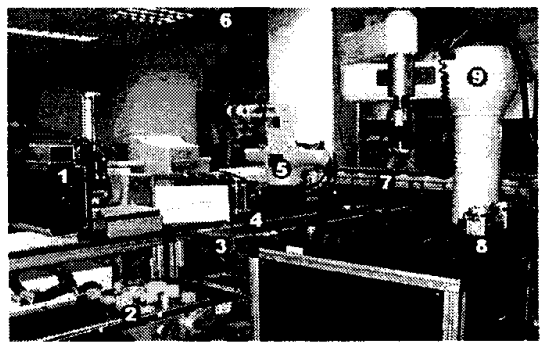

Figure 7: Two-robot assembly cell. 1: Part loader, 2 : Parts, 3 : Conveyor belt, 4 : Optical sensors, 5 : CRS robot arm, 6 : Overhead CCD camera, 7 : rotatory buffer, 8 : Grippers of Adept, 9 : Adept robot arm.

in equation (5)and(7). Relatively, its arcs can be labeled with the elements $M_{i j}$ of relationship matrix $M$, the impact function $\Phi_{i}$, and weighting parameters $\alpha_{1}, \cdots, \alpha_{N}$. With the negotiation network we proposed, the problem of multi-objective can be translated into a dual problem. It is to balance the flow for each arc in the network. There is a heuristic algorithm for the dual problem that can be summarized in the following steps. Notice that the multi-objective negotiation process coordinates with the approach of model operation per iteration $i$.

(1) Give an objective $f(R)$. According to the objective $f(R)$, tune the value of weighting parameters $\alpha_{1}, \cdots, \alpha_{N}$

(2) Check each node $O R_{j}$, examine the input stream and output stream of node $O R_{j}$, i.e., calculating the ratio of the values $\prod_{j} \frac{\sum_{i} M_{i j} C V_{i}}{\alpha_{j}}$. If the result is too large, then modify $R$.

\section{Simulation Verification on An Exam- ple}

\subsection{Example Description}

In this paper, several simulations on an example are performed to verify our proposed evaluation tool. In our laboratory, we have a two-robot assembly cell shown in Fig. 7, that is dedicated to assemble various types of mechanical parts sent serially into the conveyor belt by the part loader. There are two products produced in our assemby cell, and each product has four parts that are assembled by the robot manipulator. Parts can be placed randomly in the pallet of the loader. The loader will load the part onto the conveyor belt one at a time on request. Each robot has different mean time between failure (MTBF) and mean time to repair (MTTR). There are three objectives to be 


\begin{tabular}{|c|c|c|c|c|c|c|}
\hline Hour & 1 & 2 & 3 & 4 & 5 & 6 \\
\hline Part1 & $\mathbf{3 6}$ & $\mathbf{3 6}$ & $\mathbf{3 5}$ & $\mathbf{3 6}$ & $\mathbf{3 6}$ & $\mathbf{3 5}$ \\
\hline Part2 & $\mathbf{3 6}$ & $\mathbf{3 6}$ & $\mathbf{3 5}$ & $\mathbf{3 6}$ & $\mathbf{3 6}$ & $\mathbf{3 5}$ \\
\hline Part3 & 35 & $\mathbf{3 5}$ & $\mathbf{3 5}$ & $\mathbf{3 5}$ & $\mathbf{3 5}$ & $\mathbf{3 5}$ \\
\hline Part4 & 35 & $\mathbf{3 4}$ & $\mathbf{3 4}$ & $\mathbf{3 5}$ & $\mathbf{3 5}$ & $\mathbf{3 4}$ \\
\hline Part5 & $\mathbf{3 5}$ & $\mathbf{3 5}$ & $\mathbf{3 4}$ & $\mathbf{3 5}$ & $\mathbf{3 5}$ & $\mathbf{3 4}$ \\
\hline Part6 & $\mathbf{3 6}$ & $\mathbf{3 5}$ & $\mathbf{3 4}$ & $\mathbf{3 5}$ & $\mathbf{3 5}$ & $\mathbf{3 4}$ \\
\hline Part7 & $\mathbf{3 6}$ & $\mathbf{3 5}$ & $\mathbf{3 5}$ & $\mathbf{3 5}$ & $\mathbf{3 5}$ & $\mathbf{3 5}$ \\
\hline Part8 & $\mathbf{3 6}$ & $\mathbf{3 5}$ & $\mathbf{3 5}$ & $\mathbf{3 5}$ & $\mathbf{3 5}$ & $\mathbf{3 5}$ \\
\hline
\end{tabular}

Table 1: Arrived parts by the end of each hour

achieved, they are cycle-time(CT), throughput(TP) and Working in process(WIP) respectively. The simulation time horizon is 6 hours. At the beginning, there are 16 initial parts in this assembly cell. The total number of arrived parts at the end of each hour is listed in Table 1. For each case, three simulation runs are performed under the dynamic and stochastic environment, by choosing three different combinations of weighting parameters $\alpha_{i}$ in the objection function at Equation (7), they are $\{4,3,3\},\{2,3,5\}$ and $\{4,2,4\}$ respectively .

\subsection{Simulation Results}

This simulation is run on a Pentium PC, and the results that we will examine are the prediction variation $V_{R}^{q}\left(P_{t}\right)$ in equation (2) and the gain of feedback $U_{q}(R)$ in equation (3). They are shown in Table 2 and Table 3. For illustration, we only present the result while the combinations of weighting parameters is $\{4,2,4\}$. We let the original planned assembly scheduling is a shortest remaining first(SRF) method, it means that robots assemble the parts or subassembly products when the parts or subassembly products have shortest remaining steps to be a final product.

If we assume the actual arriving time of part in the pallet and the processing time of robot are random numbers with uniform distribution (minimum $=0.0093$, maximum $=0.0135$ ) and exponential distribution $($ mean $=30)$ respectively, then we have the mean value of performance prediction for applying the queuing theorem. However, the runs are performed under the dynamic and stochastic environment, we have the mean value for using the method of diagnosed prediction. From these results, the prediction diagnosis will work properly within the stochastic and state-dependent environment.

The SRF method is good for our assembly cell. However, we can observe that the simulation and prediction based optimization can improve all the above criteria in the log run, i.e., we have shorter mean value of cycle time, higher mean value of throughput and lower mean value of WIP. With optimization based scheduling however, the WIP is stabilized and lower than those corresponding to the heuristic dispatching rules. The results also show that the proposed on-line evaluation tool can work properly.

\begin{tabular}{|c|c|c|c|c|c|c|}
\hline Hour & 1 & 2 & 3 & 4 & 5 & 6 \\
\hline Queuing(TH) & 0.1002 & 0.0987 & 0.0992 & 0.102 & 0.102 & 0.0993 \\
\hline DPRE(TH) & 0.01 & 0.01 & 0.01 & 0.01 & 0.00993 & 0.0993 \\
\hline finsi(TH) & 0.0109 & 0.0109 & 0.011 & 0.011 & 0.011 & 0.011 \\
\hline$V_{S A F}^{T H}$ & 0.0009 & 0.0008 & 0.001 & 0.001 & 0.00167 & 0.00187 \\
\hline Queuing(CT) & 89.7 & 93 & 91.4 & 89.6 & 100 & 89.4 \\
\hline DPRE(CT) & 91.6 & 92.6 & 90.6 & 90.6 & 92 & 9 \\
\hline final(CT) & 91 & 91 & 90.4 & 90.4 & 90.4 & 90.3 \\
\hline$V_{S R F}^{C T}$ & 0.6 & 1.6 & 0.2 & 0.2 & 1.6 & 0.7 \\
\hline Queuing (WIP) & 1.835 & 1.952 & 1.842 & 1.861 & 1.842 & 1.86 \\
\hline DPRE(WIP) & 1.852 & 1.842 & 1.852 & 1.852 & 1.835 & 1.835 \\
\hline final(WIP) & 1.832 & 1.814 & 1.847 & 1.847 & 1.842 & 1.845 \\
\hline$V_{S R E}^{W M P}$ & 0.02 & 0.028 & 0.005 & 0.005 & 0.007 & 0.01 \\
\hline
\end{tabular}

Table 2: Performance Variation

\begin{tabular}{|c|c|c|c|c|c|c|}
\hline Hour & 1 & $\overline{2}$ & $\overline{3}$ & 4 & 5 & 6 \\
\hline SPOPT(CT) & 88 & 85.2 & 84.4 & 83.97 & 84.4 & 81.3 \\
\hline SRF (CT) & 91 & 91 & 90.4 & 90.4 & 90.4 & 90.3 \\
\hline$U_{C T}(S R F)$ & 0.96 & 0.93 & 0.93 & 0.92 & 0.93 & 0.90 \\
\hline SPOPT(TH) & 0.012 & 0.014 & 0.012 & 0.0126 & 0.0131 & 0.0149 \\
\hline SRF(TH) & 0.0109 & 0.0109 & 0.011 & 0.011 & 0.011 & 0.011 \\
\hline$\frac{S T H(S R F)}{U_{T H}(S)^{\prime}}$ & $\frac{0.010}{0.91}$ & 0.77 & 0.91 & $\frac{0.87}{0.87}$ & $\frac{0.02}{0.83}$ & $\frac{0.013}{0.73}$ \\
\hline SPOPT(WIP) & 1.8 & 1.714 & 1.747 & 1.74 & 1.72 & 1.79 \\
\hline SRF(WIP) & 1.832 & 1.814 & 1.847 & 1.847 & 1.842 & 1.845 \\
\hline$U_{W L P(S R F)}$ & 0.98 & 0.94 & 0.94 & 0.94 & 0.93 & 0.96 \\
\hline
\end{tabular}

Table 3: Gain of feedback

\section{Concluding Remarks}

This paper proposes a new on-line evaluation tool wherein original planned control policy designed by engineers will be evaluated before issued. A new optimal control rule or schedule derived from the original planned one will be given. We also analyze the multiobjective problem by a negotiation net. The results show the intelligence and efficiency of the proposed on-line evaluation tool.

\section{References}

[1] Robert and Alan, "Performance evaluation of automated manufacturing systems using generalized stochastic Petri Nets," IEEE Transactions on Robotics and Automation, Vol. 6, No. 6, pp. 621-639, DEC 1990.

[2] Samir M. Koriem and L. M. Patnaik, "A generalized stochastic high-level Petri Net model for performance analysis," Journal of Systems Software, pp. 247-265,36, 1997.

[3] T. C. E. Cheng, "Optimal due-date assignment in a job shop," Journal of Prod. Res., Vol. 24, No. 3, pp. 503-515, 1986.

[4] Alexandre Dolgui and Dmitry Ofitserov, "A stochastic method for discrete and continuous optimization in manu facturing systems," Journal of Intelligent Manufacturing, pp. 405-413, August 1997 .

[5] Jian Yang and Tsu-Shuan Chang, "Multiobjective scheduling for IC sort and test with a simulation tested," IEEE Transactions on Semiconductor Manufacturing, Vol. 11, No. 2, pp. 304-315, MAY 1998

[6] Shouju Ren, Xiao Dong Zhang and Xi Ping Zhang, "A new generation of decision support system for advanced manufacturing enterprises," Journal of Intelligent Manufacturing, pp. 335-343, August 1997

[7] Paul P.M. Stoop and J. Will M. Bertrand, "Performance prediction and diagnosis in two production departments," Journal of Integrated Manufacturing, pp. 335-343, August 1997. 